Arch. Tierz., Dummerstorf 47 (2004) 4, 387-395

Aus dem Institut für Tierzucht und Tierhaltung mit Tierklinik der Martin-Luther-Universität Halle-Wittenberg ${ }^{1}$ der FAL Mariensee, Braunschweig-Völkenrode ${ }^{2}$ und der Universität Ljubljana, Slowenien ${ }^{3}$

MONIKA WENSCH-DORENDORF ${ }^{1}$, NORBERT MIELENZ ${ }^{1}$, EILDERT GROENEVELD ${ }^{2}$, MILENA KOVAC ${ }^{3}$ und LUTZ SCHÜLER ${ }^{1}$

\title{
Varianzkomponentenschätzung unter Berücksichtigung von Dominanz an simulierten Reinzuchtlinien
}

\begin{abstract}
Title of the paper: Estimation of variance components under dominance with simulated purebred lines A stochastic simulation based on a gene model was used to investigate the estimation of variance with dominance and additive animal models. For a heritability in broad sense of 0.5 three ratios of dominance variance (5, 10 and 25\%) on the phenotypic variance were investigated under different degrees of dominance. No additionally biased estimations of the variance components as consequence of different dominance degrees were found. By using the dominance model for random mating as well as for selection the differences between true parameters and estimation values were small for all dominance degrees and ratios of dominance variance. Small, but significantly, differences can be explained by the change of the allele frequencies over the generations due to the influence of selection. By using the additive animal model, that ignores the dominance relationship, for high ratios of the dominance variance (25\% or greater) important biased estimations of the variances were observed. For dominance ratios of 5\% no significantly overestimation of the additive variances with the reduced model were found under selection and random mating.
\end{abstract}

Key Words: dominance, stochastic simulation, variance estimation

\section{Zusammenfassung}

Mit Hilfe von stochastischer Simulation wurde unter Verwendung eines Genmodells die Schätzung der Varianzkomponenten unter Verwendung eines Dominanzmodells und eines additiven Tiermodells untersucht. Bei einer Heritabilität im weiteren Sinn von 0,5 wurden Dominanzvarianzanteile von 5, 10 und 25\% für unterschiedliche Dominanzgrade (partielle, vollständige und Überdominanz) untersucht. Eine zusätzliche Verzerrung bei der Schätzung der Varianzkomponenten als Folge der unterschiedlichen Dominanzgrade konnte nicht nachgewiesen werden. Unter Verwendung des Dominanzmodells fallen sowohl bei den Zufalls- als auch bei den Selektionslinien die jeweiligen Unterschiede zwischen Schätzung und Vorgabe für die Varianzkomponenten für alle Dominanzgrade und Dominanzanteile relativ gering aus. Geringe, aber signifikante, Unterschiede zwischen geschätzter und vorgegebener Dominanzvarianz, lassen sich zumindest für die Selektionslinien auf die gerichtete Änderung der Allelfrequenzen als Folge der Selektion zurückführen. Bei Verwendung des additiven Tiermodells, welches die Dominanzverwandtschaft ignoriert, ergaben sich nur für hohe $\mathrm{d}^{2}$-Werte (25\% oder größer) stark verzerrte Schätzungen für die additiv-genetische Varianz über alle Dominanzgrade hinweg. Für Dominanzanteile von 5\% wurden keine signifikanten Überschätzungen der additiven Varianzen mit dem reduzierten Modell sowohl für Zufallspaarung als auch für Selektion gefunden.

Schlüsselwörter: Dominanz, stochastische Simulation, Varianzkomponenten

1 . Einleitung

Die Zuchtwertschätzung bei Nutztieren beruht gewöhnlich auf additiven Modellen (SCHÜLER u.a., 2001), wobei vorausgesetzt wird, dass mögliche Interaktionen zwischen verschiedenen Allelen an einem Genort (Dominanz) und Interaktionen zwischen den Allelen verschiedener Loci (Epistasie) ignoriert werden können. Aufgrund der ständigen Weiterentwicklung der statistischen Methoden und Computeralgorithmen 
insbesondere bei der Inversion der Dominanz-Verwandtschaftsmatrix wurde die genetische Berechnung von nichtadditiven Effekten bei großen Datensätzen möglich (HOESCHELE und VAN RADEN, 1991; MISZTAL u.a., 1995). Dabei erfordert die Schätzung der Dominanzvarianz mit Tiermodellen neben einem großen Stichprobenumfang (VAN RADEN, 1992; MIELENZ und SCHÜLER, 2004) eine Schätzmethode wie REML (PATTERSON und THOMPSON, 1971), welche invariant gegenüber Verzerrungen, hervorgerufen durch Selektion, ist.

Die Erweiterung von additiven Tiermodellen um Dominanzeffekte kann zu einer deutlichen Erhöhung der Genauigkeit bei der Schätzung genetischer Parameter beitragen (vgl. z.B. MISZTAL u.a., 1997, VERONA u.a., 1998, MIELENZ u.a. 2003)

In einer Simulationsstudie untersuchten NORRIS u.a. (2002) den Einfluss unterschiedlicher Anteile von Vollgeschwisterfamilien (zwischen 2 und $100 \%$ ) bei gleichzeitiger Variation der Dominanzvarianz an der genetischen Varianz (5, 20 und $50 \%$ ) bzw. an der Gesamtvarianz (2,5, 10 und $25 \%$ ). Bei Verwendung des einfachen VaterMutter-Modells, erweitert um Dominanzeffekte, bedingt durch die Interaktion zwischen Vater- und Mutterkomponente, zeigten die Autoren, dass die Genauigkeit der Schätzungen für die Dominanzvarianzen neben dem Anteil der Dominanzvarianz an der Gesamtvariabilität in der Population vom Grad der Dominanzverwandtschaft zwischen den Tieren abhängig ist. Ebenfalls mit einer Simulationsstudie untersuchten DUANGJINDA u.a. (2001b) den Einfluss von Selektion auf die Schätzung der Dominanzvarianz bei Verwendung von 3 Schätzmethoden (Gibbs sampling, REML, Methode R). Die Erzeugung der simulierten Datensätze über 5 Generationen erfolgte mit dem infinitesimalen Modell wie bei DUANGJINDA u.a. (2001a) beschrieben. Drei Selektionsvarianten wurden analysiert 1) zufällig 2) nach Phänotyp über die Vergleichsgruppen hinweg und 3) basierend auf Zuchtwerten geschätzt mit der BLUPMethode. Die Autoren konnten Bedingungen an die Auswahl der Elterntiere formulieren, so dass die numerisch weniger aufwendigere Methode R (REVERTER u.a., 1994) eine Alternative zur Schätzung der Dominanzvarianz mit REML und Bayesian Analysen darstellt. In vorliegender Arbeit erfolgte die Erzeugung der simulierten Datensätze analog zu den Arbeiten von De BOER u.a. (1992), BAUMUNG und SÖLKNER (1998); UIMARI und GIBSON (1998) mit Hilfe eines Genmodells. Auf Genmodellen basierende stochastische Simulationsstudien besitzen den Vorteil, dass

nichtadditive Vererbung an den Loci, Änderungen der Allelfrequenzen und genetische Drift, hervorgerufen durch die endliche Populationsgröße, in ihren Auswirkungen auf die Schätzung der genetischen Parameter berücksichtigt werden können. Ziel dieser Studie war es a) zu untersuchen welche Verzerrungen bei der Varianzkomponentenschätzung (VKS) auftreten, falls die Dominanzeffekte bei den genetischen Auswertungen ignoriert werden und b) die Genauigkeit der VKS bei variierendem Dominanzgrad an den Loci und unterschiedlichen Anteilen der Dominanzvarianz an der Gesamtvarianz zu quantifizieren.

2. Material und Methode

2.1 Simulationsprogramm und -modell

Die Erzeugung der simulierten Datensätze erfolgte mit dem Programm „rksim“, welches Populationsstrukturen und Selektionsstrategien abbilden kann, die bei der Zweili- 
nienkreuzung von Schweinen zum Einsatz kommen. Die phänotypischen Werte $y_{k}$ der Basispopulation werden mit folgendem Modell erzeugt:

(1) $y_{k}=\mu+g_{k}+e_{k} \quad$ mit $\quad g_{k}=\sum_{j=1}^{N} g_{j k}$

Hierbei sind:

$g_{j k}=$ genotypischer Effekt von Tier $k$ am Locus $j$ (mit $g_{j k} \in\{-\mathrm{a}, \mathrm{d}, \mathrm{a}\}$, wobei -a für den homozygot negativen Genotyp $\mathrm{A}_{1} \mathrm{~A}_{1}$, a für den homozy$N=$ Anzahl der Genorte/Loci

$g_{k}=$ genetischer Effekt des Tieres $k$

$\mu \quad=$ Populationsmittel (Ausgangsniveau der Population)

$e_{k} \quad=\quad$ zufälliger Resteffekt (mit $e_{k} \approx N\left(0, \sigma_{e}^{2}\right)$ )

In dieser Arbeit steht ausschließlich die genetische Auswertung von simulierten Reinzuchtlinien mit einem Tiermodell bei Berücksichtigung von Dominanz im Vordergrund. Das verwendete Simulationsprogramm erzeugt neben den zwei Reinzuchtlinien auch noch Kreuzungsnachkommen (mit Vätern aus Reinzuchtlinie 1 und Müttern aus Reinzuchtlinie 2), deren Leistungen wahlweise bei der Selektion Berücksichtigung finden können. Somit benötigt das Simulationsprogramm für die Festlegung der Populationsstrukturen noch die nachfolgenden Informationen:

- Zahl der aufeinander folgenden Generationen, in denen ein Tier eingesetzt werden kann.

- Anzahl der Elterntiere/-paare in den Reinzuchten.

- Anzahl der Elterntiere/-paare zur Erzeugung der Kreuzungsnachkommen.

- Anzahl weiblicher und männlicher Nachkommen.

- Selektion innerhalb der Nachkommen (positiv, negativ, zufällig) nach Geschlechtern getrennt.

- Einbeziehung von Leistungsinformationen (nur Reinzuchtleistungen, nur Kreuzungsleistungen oder Berücksichtigung aller Leistungsinformationen aus Reinzucht und Kreuzung).

- Anpaarungsverhältnis (in Reinzucht und Kreuzung).

- Anzahl Generationen und Anzahl Wiederholungen für die Simulation.

Für die populationsgenetischen Parameter in (1) werden noch folgende Vorgaben benötigt:

Anzahl Loci $(\mathrm{N})$, additiver Effekt (a) und Dominanzgrad ( $\mathrm{f}=\mathrm{d} / \mathrm{a}$ ) pro Locus, Allelfrequenz von $\mathrm{A}_{2}$ für die geraden $\left(\mathrm{p}_{\mathrm{g}}\right)$ und die ungeraden Loci $\left(\mathrm{p}_{\mathrm{u}}\right)$ separat für jede Reinzuchtlinie, $\mathrm{h}^{2}$ im weiteren Sinn für alle 3 Linien.

\subsection{Genetische Parameter für die Simulation}

Die nachfolgende Tabelle 1 enthält die Parametersätze für die Genmodellvarianten, welche bei der Simulation zur Anwendung kamen.

Um den Annahmen des infinitesimalen Modells genügen zu können, wurden für die genetische Merkmalsausprägung 1000 unabhängige Loci unterstellt. Der Dominanzgrad an den Loci variierte zwischen 0,4 und 1,5. Die Allelfrequenzen in der Mutterli- 
nie (Linie 2) entsprachen für das gewünschte Allel $A_{2}$ der Folge 0,9-0,4-0,9-0,4-0,9... während in der Vaterlinie (Linie 1) die an allen Genorten konstante Allelfrequenz so gewählt wurde, dass sich ein vorgegebener Dominanzgrad einstellte (vgl. Tab. 1). Bei gegebenem $\mathrm{h}^{2}$-Wert erfolgte durch entsprechende Wahl der genotypischen Effekte a und d eine Standardisierung der phänotypischen Varianz in der Vaterlinie auf den Wert Eins. Die Umweltvarianzen in den Reinzuchten und in der Kreuzung wurden so angepasst, dass die entsprechenden Heritabilitäten (im weiteren Sinn) die Werte 0,5; 0,4 und 0,35 annahmen. Um den möglichen Einfluss des Anteils der Dominanzvarianz an der Gesamtvarianz zu untersuchen, wurden Datensätze für Dominanzanteile von 25, 10 und 5\% erzeugt. Die drei unterstellten Dominanzanteile wurden zusätzlich für 3 Konstellationen des Dominanzgrades (partielle, vollständige und Überdominanz) geprüft.

Tabelle 1

Vorgabewerte (genotypische Effekte und Allelfrequenzen) für die untersuchten Genmodellvarianten sowie zugehörige genetische Varianzen berechnet mit N=1000 (Genotypic effects and allele frequencies for the gene models with corresponding variances)

\begin{tabular}{|c|c|c|c|c|c|c|c|c|}
\hline \multirow[b]{2}{*}{ Nr. } & \multicolumn{3}{|c|}{ Varianzen } & \multicolumn{2}{|c|}{ genotypische Effekte } & \multirow{2}{*}{$\begin{array}{c}\text { Linie } 1 \\
\mathrm{p}_{\mathrm{u}}=\mathrm{p}_{\mathrm{g}}\end{array}$} & \multicolumn{2}{|c|}{ Linie2 } \\
\hline & $\sigma_{a}^{2}$ & : & $\sigma_{d}^{2}$ & $\mathrm{a}$ & $\mathrm{d}$ & & $\mathrm{p}_{\mathrm{u}}$ & $\mathrm{pg}_{\mathrm{g}}$ \\
\hline \multicolumn{9}{|c|}{ Vollständige Dominanz } \\
\hline 1 & 0,25 & : & 0,25 & 0,03556 & 0,03556 & 0,6667 & 0,9 & 0,4 \\
\hline 2 & 0,40 & : & 0,10 & 0,02250 & 0,02250 & 0,3333 & 0,9 & 0,4 \\
\hline 3 & 0,45 & : & 0,05 & 0,02377 & 0,02377 & 0,1818 & 0,9 & 0,4 \\
\hline \multicolumn{9}{|c|}{ Überdominanz } \\
\hline 4 & 0,25 & $:$ & 0,25 & 0,02112 & 0,03168 & 0,4801 & 0,9 & 0,4 \\
\hline 5 & 0,40 & : & 0,10 & 0,01858 & 0,02787 & 0,2343 & 0,9 & 0,4 \\
\hline 6 & 0,45 & $:$ & 0,05 & 0,02126 & 0,03189 & 0,1270 & 0,9 & 0,4 \\
\hline \multicolumn{9}{|c|}{ Partielle Dominanz } \\
\hline 7 & 0,25 & : & 0,25 & 0,07142 & 0,05952 & 0,8423 & 0,9 & 0,4 \\
\hline 8 & 0,40 & : & 0,10 & 0,04089 & 0,02405 & 0,7052 & 0,9 & 0,4 \\
\hline 9 & 0,45 & : & 0,05 & 0,02862 & 0,01431 & 0,4456 & 0,9 & 0,4 \\
\hline 10 & 0,45 & : & 0,05 & 0,03454 & 0,01502 & 0,6207 & 0,9 & 0,4 \\
\hline
\end{tabular}

\subsection{Struktur der Population}

Die Simulation erfolgte mit 1280 Elternpaaren pro Generation bei einem Anpaarungsverhältnis von 1:16 unter der Annahme von nicht überlappenden Generationsintervallen. Pro Elternpaar wurden 2 männliche und 4 weibliche Nachkommen erzeugt. Sowohl zufällige Auswahl als auch Zuchtwertschätzung ausschließlich bei Verwendung von Reinzuchtinformationen (Eigen-, Vollgeschwister- und Halbgeschwisterleistungen) zur Selektion von 80 Vätern und 1280 Müttern auf jeder Generation wurden untersucht. Die Simulation erstreckte sich über 2 Generationen, wobei für die 1280 männlichen und die 1280 weiblichen Basistiere auf Generation Null ebenfalls phänotypische Leistungen erzeugt wurden. Folglich standen für die Schätzung der Varianzkomponenten in Linie 1 insgesamt $2 \cdot 1280+2 \cdot 6 \cdot 1280=17920$ Datensätze zur Verfügung. Die Schätzung der Varianzkomponenten mit einem rein additiven Tiermodell (reduziertes, unvollständiges Modell) und mit einem um Dominanzeffekte erweitertes Tiermodell (vollständiges Modell) erfolgte mit dem Programm VCE5.0 (KOVAC und GROENEVELD, 2002) unter Verwendung der REML-Methode. 


\section{Ergebnisse}

Nachfolgend sind die geschätzten Varianzkomponenten (jeweils gemittelt über 50 Wiederholungen) aufgeführt. Die Angabe der Ergebnisse beschränkt sich auf die Vaterlinie (Linie 1), da für diese die phänotypische Varianz auf Eins standardisiert wurde und somit die Schätzwerte für unterschiedliche Dominanzgrade und Dominanzanteile besser vergleichbar sind. In den Tabellen 2 und 3 sind jeweils die Ergebnisse mit und ohne Berücksichtigung von Dominanz im Schätzmodell angegeben. Zusätzlich enthalten beide Tabellen den Schätzfehler, also den Quotienten aus Standardfehler berechnet mit den Beobachtungen pro Wiederholung und der Wurzel aus der Anzahl Wiederholungen. Folglich wird es möglich (z.B. bei Annahme von Normalverteilung) ein Konfidenzintervall für die geschätzten Varianzen abzuleiten, so dass sich Signifikanzaussagen treffen lassen.

\subsection{Zufallspaarung}

Die Ergebnisse für Zufallspaarung bei einem Dominanzvarianzanteil von 25, 10 und 5 $\%$ an der phänotypischen Gesamtvarianz für vollständige und Überdominanz sind nachfolgend in den Tabelle 2 aufgelistet. Die Schätzungen für die additiv-genetische Varianz mit dem vollständigen Modell innerhalb der Zufallslinien lieferten sehr gute Übereinstimmungen mit den Vorgabewerten von 0,25, 0,40 und 0,45. Die prozentuale Abweichung zwischen Schätzung und Vorgabe liegt zwischen 0,1 und 1,52 \%. Für die geschätzten Dominanzvarianzen zeigten sich ebenfalls geringe Abweichungen von den Vorgaben, die allerdings für $\mathrm{d}^{2}$-Werte von 25\% signifikant ausfielen. Eine Ursache könnte sein, dass die Allelfrequenzen im Mittel über die Generationen hinweg konstant bleiben, aber bedingt durch die endliche Populationsgröße zusätzlich Variabilität über die Loci zeigen.

Tabelle 2

Mit 50 Wiederholungen geschätzte additive, dominanzbedingte und phänotypische Varianz ( \pm Schätzfehler ) im Tiermodell (mit und ohne Dominanz ) bei Zufallspaarung (Estimation of additive, dominance and phenotypic variance based on 50 replications by using an animal model with and without dominance under random mating)

\begin{tabular}{c|c|ccc|cc}
\hline \multirow{2}{*}{$\mathrm{f}=\mathrm{d} / \mathrm{a}$} & \multirow{2}{*}{$\mathrm{d}^{2}(\%)$} & $\hat{\sigma}_{a}^{2} \pm s$ & $\hat{\sigma}_{d}^{2} \pm s$ & $\hat{\sigma}_{p}^{2} \pm s$ & $\hat{\sigma}_{a}^{2} \pm s$ & $\hat{\sigma}_{p}^{2} \pm s$ \\
\cline { 2 - 7 } & 25 & $0,254 \pm 0,0022$ & $0,231^{*} \pm, 0049$ & $1,009^{*} \pm, 0020$ & $0,290^{*} \pm, 0019$ & $0,999 \pm 0,0019$ \\
1,5 & 10 & $0,398 \pm 0,0029$ & $0,102 \pm 0,0043$ & $1,004^{*} \pm, 0017$ & $0,410^{*} \pm, 0025$ & $0,998 \pm 0,0017$ \\
& 5 & $0,446 \pm 0,0026$ & $0,054 \pm 0,0044$ & $0,999 \pm 0,0017$ & $0,452 \pm 0,0024$ & $0,996 * \pm, 0017$ \\
\hline \multirow{3}{*}{1,0} & 25 & $0,253 \pm 0,0027$ & $0,240 * \pm, 0045$ & $1,009 * \pm, 0020$ & $0,291^{*} \pm, 0025$ & $0,999 \pm 0,0019$ \\
& 10 & $0,397 \pm 0,0029$ & $0,094 \pm 0,0040$ & $1,003 \pm 0,0020$ & $0,409 * \pm, 0028$ & $0,998 \pm 0,0020$ \\
& 5 & $0,446 \pm 0,0029$ & $0,051 \pm 0,0042$ & $1,000 \pm 0,0018$ & $0,451 \pm 0,0027$ & $0,997 \pm 0,0018$ \\
\hline
\end{tabular}

Mit *) markierte Werte weisen signifikante Abweichungen vom Vorgabewert für $\alpha=0,05$ auf.

Der Grad der Dominanz als auch der Anteil der Dominanzvarianz an der Gesamtvarianz scheinen bei der Schätzung der additiv-genetischen Varianz keinen Einfluss zu besitzen. Wird hingegen mit dem unvollständigen Modell, d.h. ohne Berücksichtigung der Dominanz, geschätzt, so wird ein deutlicher Einfluss des Dominanzanteiles sichtbar. Wie zu erwarten, weichen die Schätzungen der additiv-genetischen Varianz umso mehr von den wahren Werten ab, je größer der Dominanzanteil an der Gesamtvarianz ist. Allerdings fällt diese Abweichung für einen Dominanzanteil von 10\% für die zwei untersuchten Dominanzgrade nur gering (aber jeweils signifikant) aus. Für einen Do- 
minanzanteil von 25\% hingegen wird der wahre Wert, in einer Größenordnung von 16,3\% für vollständige Dominanz und 15,8\% für Überdominanz, deutlich überschätzt.

\subsection{Selektion}

Die Ergebnisse für Selektion bei einem Dominanzvarianzanteil von 25, 10 und 5 \% an der phänotypischen Gesamtvarianz für unterschiedliche Dominanzgrade sind nachfolgend in Tabelle 3 dargestellt.

Tabelle 3

Mit 50 Wiederholungen geschätzte additive, dominanzbedingte und phänotypische Varianz ( \pm Schätzfehler ) im Tiermodell (mit und ohne Dominanz ) bei Selektion (Estimation of additive, dominance and phenotypic variance based on 50 replications by using an animal model with and without dominance under selection)

\begin{tabular}{c|c|ccc|cc}
\hline \multirow{2}{*}{$\mathrm{f}=\mathrm{d} / \mathrm{a}$} & \multirow{2}{*}{$\mathrm{d}^{2}(\%)$} & $\hat{\sigma}_{a}^{2} \pm s$ & $\hat{\sigma}_{d}^{2} \pm s$ & $\hat{\sigma}_{p}^{2} \pm s$ & $\hat{\sigma}_{a}^{2} \pm s$ & $\hat{\sigma}_{p}^{2} \pm s$ \\
\cline { 3 - 7 } & 25 & $0,247 \pm 0,0019$ & $0,227^{*} \pm, 0051$ & $1,004^{*} \pm, 0017$ & $0,270^{*} \pm, 0019$ & $0,992^{*} \pm, 0017$ \\
1,5 & 10 & $0,397 \pm 0,0025$ & $0,103 \pm 0,0038$ & $1,006^{*} \pm, 0016$ & $0,404 \pm 0,0023$ & $0,998 \pm 0,0016$ \\
& 5 & $0,448 \pm 0,0030$ & $0,050 \pm 0,0038$ & $1,006^{*} \pm, 0019$ & $0,450 \pm 0,0029$ & $1,002 \pm 0,0019$ \\
\hline & 25 & $0,247 \pm 0,0024$ & $0,231^{*} \pm, 0045$ & $1,001 \pm 0,0018$ & $0,271^{*} \pm, 0024$ & $0,988^{*} \pm, 0017$ \\
1,0 & 10 & $0,396 \pm 0,0026$ & $0,093 \pm 0,0038$ & $1,004^{*} \pm, 0018$ & $0,402 \pm 0,0024$ & $0,997 \pm 0,0018$ \\
& 5 & $0,447 \pm 0,0029$ & $0,052 \pm 0,0035$ & $1,005^{*} \pm, 0019$ & $0,450 \pm 0,0028$ & $1,001 \pm 0,0019$ \\
\hline $1 / 1,2$ & 25 & $0,247 \pm 0,0025$ & $0,216^{*} \pm, 0045$ & $0,992^{*} \pm, 0019$ & $0,270^{*} \pm, 0024$ & $0,980^{*} \pm, 0019$ \\
$1 / 1,7$ & 10 & $0,394 \pm 0,0030$ & $0,087^{*} \pm, 0039$ & $0,994^{*} \pm, 0020$ & $0,400 \pm 0,0029$ & $0,988^{*} \pm, 0020$ \\
$1 / 2,0$ & 5 & $0,446 \pm 0,0025$ & $0,047 \pm 0,0042$ & $0,999 \pm 0,0016$ & $0,448 \pm 0,0025$ & $0,995^{*} \pm, 0016$ \\
$1 / 2,3$ & 5 & $0,448 \pm 0,0025$ & $0,050 \pm 0,0039$ & $0,999 \pm 0,0018$ & $0,450 \pm 0,0025$ & $0,995^{*} \pm, 0018$ \\
\hline
\end{tabular}

Die Selektion erstreckte sich lediglich über 2 Generationen, um den Einfluss von Inzucht und die Änderung der Allelfrequenzen möglichst gering zuhalten. Für die Schätzungen der additiv-genetischen Varianz der Selektionslinien zeigt sich ein ähnliches Bild wie unter Zufallspaarung. Wird mit dem vollständigen Modell geschätzt, so ergibt sich eine sehr gute Übereinstimmung mit den wahren Werten für alle Dominanzgrade und Dominanzanteile. Die prozentuale Abweichung liegt nur zwischen 0,05 und 1,45 $\%$. Allerdings wird die additiv-genetische Varianz für alle zehn in Tabelle 3 aufgelisteten Varianten gering, aber nicht signifikant, unterschätzt. Dagegen ergab sich für die Dominanzvarianz bei $\mathrm{d}^{2}$-Werten von $25 \%$ für alle untersuchten Dominanzgrade eine geringe, aber generell signifikante Unterschätzung. Betrachtet man die Ausgangsfrequenz des gewünschten Allels bei signifikanter Unterschätzung der Dominanzvarianz, so lag diese in sämtlichen Fällen über 0,5. Durch den Einfluss von Selektion kommt es zwangsläufig zu einer Erhöhung der Frequenz des gewünschten Allels. Gemäß der Theorie für Einlocus-Modelle (SCHÜLER u. a., 2001) ist für Ausgangsfrequenzen größer als 0,5 bei Frequenzerhöhung ein Absinken der Dominanzvarianz in der Population zu erwarten.

Im Mehrlocus-Modell baut sich zusätzlich, bedingt durch den Selektionsprozess, über die Generationen hinweg auch bei Annahme von ungekoppelten Loci ein Gametenphasenungleichgewicht auf. Betrachtet man im Multilocus-Modell zwei zufällig herausgegriffene Genorte $A$ und $B$ mit jeweils zwei Allelen $A_{1}, A_{2}$ und $B_{1}, B_{2}$ so kann das Kopplungsungleichgewicht mit Hilfe der Gametenfrequenzen $P\left(A_{i} B_{j}\right)$ durch den folgenden Koeffizienten beschrieben werden (CROW und KIMURA, 1970). 


$$
D_{A B}=P\left(A_{1} B_{1}\right) \cdot P\left(A_{2} B_{2}\right)-P\left(A_{1} B_{2}\right) \cdot P\left(A_{2} B_{1}\right)
$$

Bei Beschränkung auf Ungleichgewicht jeweils nur an Paaren von Genorten ergibt sich bei Abwesenheit von Epistasie im biallelen Mehrlocusfall für die additive Varianz und die Dominanzvarianz die Darstellung (LYNCH und WALSH, 1997):

$$
\begin{aligned}
& \sigma_{a}^{2}=2 \cdot \sum_{i=1}^{N} p_{i} \cdot\left(1-p_{i}\right) \cdot \alpha_{i}^{2}+4 \cdot \sum_{i<j} \alpha_{i} \cdot \alpha_{j} \cdot D_{i j} \\
& \sigma_{d}^{2}=4 \cdot \sum_{i=1}^{N}\left[d_{i} \cdot p_{i} \cdot\left(1-p_{i}\right)\right]^{2}+8 \cdot \sum_{i<j} d_{i} \cdot d_{j} \cdot D_{i j}^{2}
\end{aligned}
$$

In (3) bezeichnet $\alpha_{\mathrm{i}}$ den durchschnittlichen Effekt einer Allelsubstitution am Locus i. Die Auswirkungen des Gametenphasenungleichgewichts auf die Aufspaltung der genetischen Varianz in additive und dominanzbedingte Varianz lässt sich im Mehrlocusfall nur schwer abschätzen, weil das Vorzeichen der Koeffizienten $D_{\mathrm{ij}}$ z.B. in Abhängigkeit von der Fitness der Genotypen und somit von der Selektion variieren kann. Sind die Koeffizienten $\mathrm{D}_{\mathrm{ij}}$ in der Mehrzahl negativ so ist ein zusätzlicher Abfall der additiven Varianz zu erwarten. Hinsichtlich der Dominanzvarianz ergibt sich bei gerichteter Dominanz (bei der Simulation wurde $\mathrm{d}_{\mathrm{i}}=\mathrm{d}>0$ für alle Loci angenommen) stets ein positiver Beitrag, der kleine $\mathrm{D}_{\mathrm{ij}}$-Werte vorausgesetzt äußert gering ausfallen wird und somit den Abfall der Dominanzvarianz bei entsprechender Änderung der Allelfrequenzen nicht kompensieren kann.

Betrachtet man die Ergebnisse für das unvollständige Modell in Tabelle 3, so werden nur für $\mathrm{d}^{2}$-Werte von $25 \%$ hochsignifikante Überschätzungen ausgewiesen. Für den Dominanzanteil von 25\% liegt die prozentuale Abweichung bei 7,97\% (partielle Dominanz mit $\mathrm{f}=1 / 1,2$ ), 8,53\% (vollständige Dominanz) und 8,06\% (Überdominanz) und ist damit geringer als bei den Zufallslinien. Bei Dominanzanteilen von 5\% und $10 \%$ liegt die prozentuale Abweichung nur zwischen 0,006 und 2,0\% und fällt damit sehr gering und nicht signifikant zu den Vorgabewerten aus.

Vernachlässigt man die Dominanzeffekte, indem die Auswertung mit einem rein additiven Tiermodell erfolgt, so sind die Dominanzeffekte bei Abwesenheit von permanenten Umwelteffekten (wie z.B. von Umwelteinflüssen, die allen Tieren einer Vollgeschwistergruppe gemeinsam sind) als Bestandteil der zufälligen Resteffekte anzusehen (MISZTAL u.a., 1995). Aufgrund der genetischen Kovarianz zwischen verwandten Tieren treten damit insbesondere zwischen den eigentlich umweltbedingten Resteffekten von Vollgeschwistern Kovarianzen auf, deren Vorhandensein im additiven Tiermodell ignoriert werden müssen. Ändern sich die Dominanzvarianzen als Folge der Selektion über die Generationen hinweg, so wird zusätzlich gegen die Voraussetzung homogener Restvarianzen verstoßen. Als Konsequenz dieser Modellverletzungen sind für alle Varianzkomponenten im Modell verzerrte Schätzungen zu erwarten, wobei die vorhandene Dominanzvarianz sowohl zum Teil in die additive Varianz als auch in die Restvarianz eingeht (MISZTAL, 1997). Bei den hier untersuchten Varianten wurde die Dominanzvarianz nur für $\mathrm{d}^{2}$-Werte von $25 \%$ nicht fast vollständig als Bestandteil der Restvarianz geschätzt. Bei Verwendung des unvollständigen Modells fließt für derartig hohe Anteile ein gewisser Teil der Dominanzvarianz in die Schätzung für die additiv-genetische Varianz ein. Zumindest für $\mathrm{d}^{2}$-Werte von $5 \%$ konnte dieser Nachteil bei der Verwendung des unvollständigen Modells sowohl bei Zufallspaarung als auch bei Selektion nicht beobachtet werden. Unter Selektion ergaben sich sogar für Dominanzanteile von 10\% keine signifikanten Überschätzungen. Diese Aus- 
sage steht im Gegensatz zu den Ergebnissen von NORRIS u.a. (2002), welche für die Konstellationen (500:500:1000); (800:200:2000) und (950:50:1000) von additiver Varianz, Dominanz- und Umweltvarianz stets eine Überschätzung der additiven Varianz mit dem reduzierten Modell fanden. Allerdings nutzten die Autoren für die Simulation das einfache Vater-Mutter-Modell wobei die Dominanzeffekte über die Interaktion zwischen Vater- und Mutterkomponente abgebildet wurde. Zur Schätzung der Varianzen mit Daten aus nur einer Generation verwendeten die Autoren die „tilde-hat“ Approximation von REML (VAN RADEN und JUNG, 1988), welche neben der Abwesenheit von Selektion eine Ursache für die abweichenden Ergebnisse darstellt. Es sei bemerkt, dass sich die Konsequenzen der Ignorierung von Dominanzeffekten im Modell nicht nur auf die Schätzung der Varianzen auswirken können sondern dass für verschiedene Tiergruppen, charakterisiert durch einen gemeinsamen Verwandtschaftsgrad, Auswirkungen auf die Zuchtwertschätzung zu erwarten sind. Eine unverzerrte Schätzung der additiven Varianz mit dem reduzierten Modell lässt noch keine Aussagen über die Qualität der Zuchtwertschätzung von Tieren, dessen Verwandtschaftskoeffizienten große nichtadditive Kovarianzen enthalten, zu.

Aufgrund der Ergebnisse, aufgelistet in den Tabellen 2 und 3, konnte eine Verzerrung bei der Schätzung der Varianzkomponenten als ausschließliche Folge der unterschiedlichen Dominanzgrade nicht nachgewiesen werden.

Bei Verwendung des additiven Tiermodells, also des unvollständigen Modells, ergaben sich nur für hohe $\mathrm{d}^{2}$-Werte (25\%) stark verzerrte Schätzungen für die additiv-genetische Varianz über alle Dominanzgrade hinweg. Für Dominanzanteile von 5\% konnten trotz Nichteinhaltung von Modellvoraussetzungen sowohl bei Zufallspaarung als auch bei Selektion keine signifikanten Verzerrungen bei der Schätzung der additiven Varianz beobachtet werden. Weiterführende Untersuchungen zum Vergleich der Zuchtwertschätzung mit Dominanzmodellen und rein additiven Modellen bei Vernachlässigung von nichtadditiven Effekten sind notwendig.

\section{Literatur}

BAUMUNG, R.; SÖLKNER, J.; ESSL, A.:

Optimization of pig crossbreeding programmes with regard to the genetic development of involved purebred populations. $49^{\text {th }}$ annual meeting of EAAP (1998) Warsaw, Poland

CROW, F.J.; KIMURA, M.:

An introduction to population genetics theory. Alpha Editions, Inc. Edina, MM. (1970)

DE BOER, I.J.M.; VAN ARENDONK, J.A.M.:

Prediction of additive and dominance effects in selected or unselected populations with inbreeding. Theor. Appl. Genet. 84 (1992), 451-459

DUANGJINDA, M.; MISZTAL, I.; BERTRAND, J.K.:

Generating data in models including direct and maternal dominance effects. J. Appl. Genet. 42 (2001a), 193-204

DUANGJINDA, M.; MISZTAL, I.; BERTRAND, J.K.:

Empirical bias of estimates by REML, Bayesian method via Gibbs Sampling and Method R under selection for additive, maternal and dominance models. J. Anim. Sci. 79 (2001b), 2991-2996

HOESCHELE, I.; VAN RADEN, P.M.:

Rapid inversion of dominance relationship matrices for noninbred populations by including sire by dam subclass effects. J. Dairy Sci. 74 (1991), 557-569

KOVAC, M.; GROENEVELD, E.; GARCIA-CORTES, L.A.:

VCE-5, a package for the estimation of dispersion parameters. Proc. $7^{\text {th }}$ WCGALP (2002) Montpellier

LYNCH, M.; WALSH, B.:

Genetics and analysis of quantitative traits. Sinauer Associates, Inc. Sunderland, MA. ?

MIELENZ, N.; KOVAC, M.; GROENEVELD, E.; PREISINGER, R.; SCHMUTZ, M.; SCHÜLER, L.: 
Genetische Parameter für Merkmale der Eiproduktion geschätzt mit additiven und Dominanzmodellen bei Legehennen. Arch. Tierz., Dummerstorf 46 (2003) 1, 77-84

MIELENZ, N.; SCHÜLER, L.:

Stichprobenumfang zur Schätzung der Dominanzvarianz bei hierarchischer Halb- und Vollgeschwisterstruktur. Arch. Tierz., Dummerstorf (2004) eingereicht

MISZTAL, I.; FERNANDO, R.L.; GROSSMANN, M.; LAWLOR, T.J.; LUKASZEWICZ, M.:

Dominance and epistatic effects in genetic evaluation of farm animals. Animal Science Papers and Reports 13 (1995) no. 4, 251-266

MISZTAL, I.:

Estimation of variance components with large-scale dominance model. J. Dairy Sci. 80 (1997), 965-974

NORRIS, D.; MAO, L.L.; COETZEE; R.J.:

Effect of population structure and underlying magnitude of dominance genetic effects on the estimation of additive and dominance genetic variances. South African Journal of Animal Science 32 (2002) 2,113-129

PATTERSON, H.D.; THOMPSON, R.:

Recovery of inter-block information when block sizes are unequal. Biometrika 58 (1971), 545-554

REVERTER, A.; GOLDEN, B.L.; BOURDON, R.M.:

Method R variance components procedure: application on the simple breeding value model. J. Anim. Sci. 72 (1994), 2247

SCHÜLER, L.; SWALVE, H.; GÖTZ, K.U.:

Grundlagen der quantitativen Genetik. Verlag Eugen Ulmer, Stuttgart (2001)

UIMARI, P.; GIBSON, J.:

Combined crossbred and purebred selection vs pureline selection for a sex limited trait in poultry. Proceedings of the $6^{\text {th }}$ WCGALP, vol. 25 (1998), 455-458, Armidale, Australia

VAN RADEN, P.M.; JUNG, Y.C.:

A general purpose approximation to Restricted Maximum Likelihood. The tilde-hat approach. J. Dairy Sci. 71 (1988), 187

VAN RADEN, P.M.; LAWLOR, T.J.; SHORT, T.H.; HOESCHELE, I.:

Use of reproductive technology to estimate variances and predict effects of gene interactions. J. Dairy Sci. 75 (1992), 2892-2901

VARONA, L.; MISZTAL, I.; BERTRAND, J.K.; LAWLOR, T.J.:

Effect of full sibs on additive breeding values under the dominance model for stature in united states holsteins. J. Dairy Sci. 81 (1998), 1126-1135

Eingegangen: 23.12.2003

Akzeptiert: 14.06.2004

Anschriften der Verfasser

Dr. MONIKA WENSCH-DORENDORF,* Dr. NORBERT MIELENZ,

Prof. Dr. habil. LUTZ SCHÜLER

Institut für Tierzucht und Tierhaltung mit Tierklinik der Martin-Luther-Universität

Halle-Wittenberg

Adam-Kuckhoff-Straße 35

D-06108 Halle

Prof. Dr. Dr. h.c. EILDERT GROENEVELD

Institut für Tierzucht und Tierverhalten Mariensee der FAL

Höltystr. 10,

D-31535 Neustadt

Prof. Dr. MILENA KOVAC, PhD

Dept. of Animal Science, Biotechnical Fakulty, University of Lubljana

Groblje 3

Sl-1230 Domzale

Slovenia

* Korrespondierender Autor 